\title{
Abelian Theorem for Generalized Mellin-Whittaker Transform
}

\author{
A. S. Gudadhe ${ }^{1}$, R. V. Kene ${ }^{2}$ \\ ${ }^{1,2}$ (Head Department of Mathematics, GVISH/Amravati, M S, India)
}

Abstract: The paper provides the Abelian and Terberian theorem for generalized Mellin-Whittaker transform. Key-words:Abeliantheorem, Generalized Mellin-Whittaker transform.

\section{Introduction:}

Integral transformation is one of the well known techniques used for function transformation. Integral transform methods have proved to be of great importance in initial and boundary value problems of partial differential equation.

Extension of some transformations to generalized functions has been done time to time and their properties have been studied by various mathematicians. However there is much scope in extending double and triple transformation to a certain class of generalized functions. Bhosale B. N. and Choudhary M. S. [1], Sharma V. D. and Gudadhe A. S. [2] has discussed double transforms. Motivated by this we have also defined a new combination of integral transforms on the spaces of generalized functions namely Mellin-Whittaker transform. Along with the definition its analyticity theorem is proved in [3], where as inversion theorem is investigated in [4]. Now in the present paper we established the abelian theorem for generalized Mellin-Whittaker transform.

In section 2 we have defined the spaces $E_{\alpha, \infty}^{\prime}$ and $E_{-\infty, w}^{\prime}$ on which Mellin transform is defined. We have given the definition of generalized Mellin Whittaker transform in section 3. Section 4 is devoted toInitial value theorem for generalized Mellin-Whittaker transform. Finally in section 5 final value theorem for generalized Mellin-Whittaker transform is established.

Notations and terminology as per Zemanian [5].

\section{Spaces $\boldsymbol{E}_{\alpha, \infty}^{\prime}$ and $\boldsymbol{E}_{-\infty, \boldsymbol{w}}^{\prime}$ :}

By $E_{b, c}(b, c$ are finite real numbers with $b<c$ ) we denote the linear space of infinitely differentiable functions $\varphi(x)$ defined on $[0, \infty]$ and such that there exist two strictly positive numbers $\zeta$ and $\zeta^{\prime}$ for which $x^{k+1-b-\zeta} \varphi^{k}(x) \rightarrow 0$ as $x \rightarrow 0+$ and $x^{k+1-c-\zeta^{\prime}} \varphi^{k}(x) \rightarrow 0$ as $x \rightarrow \infty$ for all $k \in N$, where $N$ is the set of non negative integers. We set $x_{+}^{s-1}=\left\{\begin{array}{ll}x^{s-1}, & x>0 \\ 0, & x<0\end{array}\right.$ so that $x_{+}^{s-1}$ belongs to $E_{b, c}$ if $b<\operatorname{Res}<c$. Put $K_{b, c}(x)=\left\{\begin{array}{rr}x^{-b}, & 0<x \leq 1 \\ x^{-c} & x \geq 1\end{array}\right.$ and $\gamma_{k, b, c}(\varphi)={ }_{x>0}^{\sup } x_{b, c} x^{k+1}\left|\varphi^{k}(x)\right|$.

$\gamma_{k, b, c}(\varphi)$ are all bounded and are seminorms, $\gamma_{0, b, c}$ is a norm. We now provide the following topology in $E_{b, c}$.

A sequence $\left\{\varphi_{j}\right\} \rightarrow 0$ in $E_{b, c}$ if and only if $\gamma_{k, b, c}\left(\varphi_{j}\right) \rightarrow 0$ for each $k \in N$.Thus $E_{b, c}$ is provided with a structure of a countable multi-normed space. Also, $E_{-\infty, c}$ is the inductive limit of $E_{b, c}$ as $b \rightarrow-\infty$. This means that a sequence $\left\{\varphi_{j}\right\} \rightarrow 0$ in $E_{-\infty, c}$ if and only if there exist a $b<c$ such that $\varphi_{j} \in E_{b, c}$ and $\left\{\varphi_{j}\right\} \rightarrow 0$ in $E_{b, c}$ (i.e. $\gamma_{k, b, c}\left(\varphi_{j}\right) \rightarrow 0$ as $\left.j \rightarrow \infty\right)$.

In a similar manner, $E_{\alpha, \infty}$ is the inductive limit of $E_{b, c}$ as $b \rightarrow \alpha, c \rightarrow \infty$ and $E_{-\infty, w}$ is the inductive limit of $E_{b, c}$ as $b \rightarrow-\infty, c \rightarrow w \cdot E_{\alpha, \infty}^{\prime}$ is the dual of $E_{\alpha, \infty}$ and $E_{-\infty, w}^{\prime}$ is the dual of $E_{-\infty, w}$.

\section{Generalized Mellin Whittaker Transform:}

For $f(x, t) \in M W_{a, b}^{\prime}$, we define distributional Mellin Whittaker transform of a function $f(x, t)$ as, $M W\{f(x, t)\}=F(s, y)=\left\langle f(x, t), x^{s-1} e^{-\frac{q}{2} y t}(y t)^{m-\frac{1}{2}} W_{k, m}(p y t)\right\rangle(3.1)$ RHS of (3.1) has meaning, for $f \in M W_{a, b}^{\prime}$ and $x^{s-1} e^{-\frac{q}{2} y t}(y t)^{m-\frac{1}{2}} W_{k, m}(p y t) \in M W_{a, b}$.

\section{Initial Value Theorem For Generalized Mellin-Whittaker Transform:}

In this section we have proved initial value theorem for generalized Mellin-Whittaker transform.

Theorem: Let $f(x, t)$ be locally integrable function with distributional derivatives w.r.to $x$ belonging to $E_{\alpha, \infty}^{\prime}$ for some real number $\alpha$ and having compact support for $x$ in $[0, a]$. Moreover

$\frac{f(x, t)}{e^{c t}}$ is absolutely integrable for $c \in R$.

$\lim _{\substack{x \rightarrow a \\ t \rightarrow 0}} \frac{f(x, t)}{\left(\log \frac{a}{x}\right)^{v} t^{\eta}}=\beta \quad \operatorname{Rev}>-1, \quad \eta>-1$. Then 
$\lim _{\substack{s \rightarrow \infty \\ y \rightarrow \infty}} \frac{s^{v+1} y^{\eta+1} F(s, y)}{a^{s}\lceil(v+1) B(m, \eta, m, k, q, p)}=\beta$ in the half plane $\operatorname{Res}>\alpha$,

where $B(m, \eta, m, k, q, p)=\frac{p^{m+\frac{1}{2}} \Gamma(m \pm m+\eta+1)}{\left[\frac{1}{2}(q+p)\right]^{m+m+\eta+1} \Gamma\left(m-k+\eta+\frac{3}{2}\right)}$

$$
\times 2 F_{1}\left[\begin{array}{c}
m+m+\eta+1, m-k+\frac{1}{2} ; \\
\frac{q-p}{q+p} \\
m-k+\eta+\frac{3}{2} ;
\end{array}\right]
$$

Proof: We prove the theorem for a restricted type of functions i.e. when $f(x, t)$ is seperable.

Let $f(x, t)=g(x) h(t)(4.1)$

Therefore we can assume that $\beta=\beta_{1}$. $\beta_{2}$, where $\beta_{1}, \beta_{2}$ are real numbers such that, property (ii) becomes

$$
\left.\begin{array}{cc}
\lim _{x \rightarrow a} \frac{g(x)}{\left(\log \frac{a}{x}\right)^{v}}=\beta_{1} & \text { Re } v>-1 \\
\lim _{t \rightarrow 0} \frac{h(t)}{t^{\eta}}=\beta_{2} & \eta>-1
\end{array}\right\},
$$

If we put $x=a e^{-V}$ then $\lim _{V \rightarrow 0} \frac{g\left(a e^{-V}\right)}{V^{v}}=\beta_{1}$

(4.2) and (4.3) $\Rightarrow \lim _{\substack{x \rightarrow a \\ t \rightarrow 0}} \frac{g(x) h(t)}{\left(\log \frac{a}{x}\right)^{v} t^{\eta}}=\beta_{1} \cdot \beta_{2}=\beta$

$\operatorname{orim}_{\substack{V \rightarrow 0 \\ t \rightarrow 0}} \frac{g\left(a e^{-V}\right) h(t)}{(V)^{v} t^{\eta}}=\beta_{1} \cdot \beta_{2}=\beta$

We know that $(M(g(x)))(s)=a^{s}\left(\operatorname{Lg}\left(a e^{-V}\right)\right)(s)$.

Also for $\eta>-1$ and $t>0$

$\int_{0}^{\infty} t^{\eta} e^{-\frac{q}{2} y t}(y t)^{m-\frac{1}{2}} W_{k, m}(p y t) d t=\frac{1}{y^{\eta+1}} B(m, \eta, m, k, q, p)$

$\int_{0}^{\infty} V^{v} e^{-s \vee} d V=\frac{\lceil(v+1)}{s^{v+1}}$ for $v>-1$

Now consider,

$$
\begin{gathered}
\mid s^{v+1} y^{\eta+1} F(s, y)-\beta\left\lceil(v+1) a^{s} B(m, \eta, m, k, q, p) \mid\right. \\
=\mid s^{v+1} y^{\eta+1} \int_{0}^{\infty} \int_{0}^{a} x^{s-1} e^{-\frac{q}{2} y t}(y t)^{m-\frac{1}{2}} W_{k, m}(p y t) g(x) h(t) d x d t-\beta_{1} \cdot \beta_{2}\left[(v+1) a^{s} B(m, \eta, m, k, q, p) \mid\right.
\end{gathered}
$$

$\because f(x, t)$ has compact support in $[0, a]$ w.r.t. $x$.

Put $x=a e^{-V} \quad \therefore x^{s-1} d x=-a^{s} e^{-s V} d V$

Also $f(x, t)=g(x) h(t)=g\left(a e^{-V}\right) h(t)$ and as $x$ varies from 0 to $a$ and $V$ varies from coto 0 .

Therefore absorbing negative sign and using (4.5), (4.6) we have LHS of (4.7) as,

$$
\begin{aligned}
& \text { LHS }=\mid s^{v+1} y^{\eta+1} \int_{0}^{\infty} \int_{0}^{a} e^{-\frac{q}{2} y t}(y t)^{m-\frac{1}{2}} W_{k, m}(p y t) a^{s} e^{-s V} g\left(a e^{-V}\right) h(t) d V d t \\
& \quad-\beta_{1} \cdot \beta_{2} s^{v+1} \int_{0}^{\infty} V^{v} e^{-s V} d V a^{s} y^{\eta+1} \int_{0}^{\infty} t^{\eta} e^{-\frac{q}{2} y t}(y t)^{m-\frac{1}{2}} W_{k, m}(p y t) d t \mid \\
& =\left|s^{v+1} y^{\eta+1} \int_{0}^{\infty} \int_{0}^{\infty} e^{-\frac{q}{2} y t}(y t)^{m-\frac{1}{2}} W_{k, m}(p y t) a^{s} e^{-s V}\left[g\left(a e^{-V}\right) h(t)-\beta_{1} \cdot \beta_{2} V^{v} t^{\eta}\right] d V d t\right| \\
& =\left|s^{v+1} y^{\eta+1} \int_{0}^{\infty} \int_{-\infty}^{\infty} e^{-\frac{q}{2} y t}(y t)^{m-\frac{1}{2}} W_{k, m}(p y t) a^{s} e^{-s V}\left[g\left(a e^{-V}\right) h(t)-\beta_{1} \cdot \beta_{2} V^{v} t^{\eta}\right] d V d t\right| \\
& \leq I_{1}+I_{2}
\end{aligned}
$$

where

$$
\begin{aligned}
& I_{1}=\left|s^{v+1} y^{\eta+1} \int_{0}^{Y} \int_{0}^{Y} e^{-\frac{q}{2} y t}(y t)^{m-\frac{1}{2}} W_{k, m}(p y t) a^{s} e^{-s V}\left[g\left(a e^{-V}\right) h(t)-\beta_{1} \cdot \beta_{2} V^{v} t^{\eta}\right] d x d t\right| \\
& I_{2}=\left|s^{v+1} y^{\eta+1} \int_{Y}^{\infty} \int_{Y}^{\infty} e^{-\frac{q}{2} y t}(y t)^{m-\frac{1}{2}} W_{k, m}(p y t) a^{s} e^{-s V}\left[g\left(a e^{-V}\right) h(t)-\beta_{1} \cdot \beta_{2} V^{v} t^{\eta}\right] d x d t\right|
\end{aligned}
$$

As $W_{k, m}(z)=O\left(z^{k} e^{-\frac{z}{2}}\right), \quad|z| \rightarrow \infty$

$$
=O\left(z^{m+\frac{1}{2}}\right), \quad|z| \rightarrow 0, m<0
$$




$$
=O\left(z^{m-\frac{1}{2}}\right), \quad|z| \rightarrow 0, m>0
$$

The function $s^{v+1} y^{\eta+1} e^{-\frac{q}{2} y t}(y t)^{m-\frac{1}{2}} W_{k, m}(p y t) a^{s} e^{-s V}$ is bounded as $s \rightarrow \infty$ and $y \rightarrow \infty$.

That is $\left|s^{v+1} y^{\eta+1} e^{-\frac{q}{2} y t}(y t)^{m-\frac{1}{2}} W_{k, m}(p y t) a^{s} e^{-s V}\right| \leq M$ as $s \rightarrow \infty$ and $y \rightarrow \infty$ for $0<x<Y$ and $0<t<Y$.

Therefore $I_{1} \leq \sup _{\substack{0<x<Y \\ 0<t<Y}}\left|M Y^{2} \| g\left(a e^{-V}\right) h(t)-\beta_{1} \cdot \beta_{2} V^{v} t^{\eta}\right|$

$\therefore$ for $\epsilon=\frac{\epsilon}{2 M Y^{2}}>0$ there exists $\delta>0$ such that

$$
|x|<\delta_{1},|t|<\delta_{2} \Rightarrow\left|g\left(a e^{-V}\right) h(t)-\beta_{1} \cdot \beta_{2} V^{v} t^{\eta}\right|<\frac{\epsilon}{2 M Y^{2}}
$$

$\therefore I_{1}<\frac{\epsilon}{2}$ ast $\rightarrow 0, V \rightarrow 0$ using (3), for all $\in>0$.

Now for $I_{2}=\left|s^{v+1} y^{\eta+1} \int_{Y}^{\infty} \int_{Y}^{\infty} e^{-\frac{q}{2} y t}(y t)^{m-\frac{1}{2}} W_{k, m}(p y t) a^{s} e^{-s V}\left[g\left(a e^{-V}\right) h(t)-\beta_{1} \cdot \beta_{2} V^{v} t^{\eta}\right] d V d t\right|$

By the property (i) $\frac{f(x, t)}{e^{c t}}$ is absolutely integrable hence $\frac{g\left(a e^{-V}\right) h(t)}{e^{c t}}$ is absolutely integrable and $I_{2}$ can be written as,

$$
I_{2}=\left|s^{v+1} y^{\eta+1} \int_{Y}^{\infty} \int_{Y}^{\infty} e^{-\frac{1}{2}(q y-2 c) t}(y t)^{m-\frac{1}{2}} W_{k, m}(p y t) a^{s} e^{-s V} e^{-c t}\left[g\left(a e^{-V}\right) h(t)-\beta_{1} \cdot \beta_{2} V^{v} t^{\eta}\right] d V d t\right|
$$

Now the function $e^{-s V} e^{-\frac{1}{2}(q y-2 c) t}(y t)^{m-\frac{1}{2}} W_{k, m}(p y t)$ is finite and continuous in $Y<T<\infty$ and it $\rightarrow 0$ as $t \rightarrow \infty$ if $\operatorname{Re}(q+p) x>2 c$.

Let the upper bound of this function be attained at the point $(V, t)=\left(r_{1}, r_{2}\right)$ and

$\int_{Y}^{\infty} \int_{Y}^{\infty}\left[g\left(a e^{-V}\right) h(t)-\beta_{1} \cdot \beta_{2} V^{v} t^{\eta}\right] d V d t=M_{1}$ say then

$I_{2} \leq M_{1}\left[e^{-s r_{1}} e^{-\frac{1}{2}(q y-2 c) r_{1}}\left(y r_{2}\right)^{m-\frac{1}{2}} W_{k, m}\left(p y r_{2}\right)\right] s^{v+1} y^{\eta+1}$

Choose $y$ sufficiently small so that r.h.s. of (4.10) becomes less than $\frac{\epsilon}{2}$.

Hence (4.8) becomes

$$
\mid s^{v+1} y^{\eta+1} F(s, y)-\beta\left\lceil(v+1) a^{s} B(m, \eta, m, k, q, p) \mid \leq I_{1}+I_{2}<\frac{\epsilon}{2}+\frac{\epsilon}{2}=\epsilon\right.
$$

Hence the theorem is proved.

\section{Final Value Theorem For Generalized Mellin-Whittaker Transform:}

In this section we established final value theorem for generalized Mellin-Whittaker transform.

Theorem: Let $f(x, t)$ be locally integrable function with all distributional derivatives w.r.to $x$ belonging to $E_{-\infty, w}^{\prime}$, for some real number $w$ and having compact support for $x$ in $[b, \infty)$. Moreover

$\frac{f(x, t)}{x^{c}}$ is absolutely integrable for some $c \in R$.

$\lim _{\substack{x \rightarrow \infty \\ t \rightarrow \infty}} \frac{f(x, t)}{\left(\log _{\frac{x}{b}}^{x}\right)^{v} t^{\eta}}=\beta \quad \operatorname{Rev}>-1, \operatorname{Re\eta }>-1$. Then

$\lim _{\substack{|s| \rightarrow 0 \\ y \rightarrow 0}} \frac{s^{v+1} y^{\eta+1} F(s, y)}{b^{s} \Gamma(v+1) B(m, \eta, m, k, q, p)}=\beta$ in the half plane Res $>\alpha$,

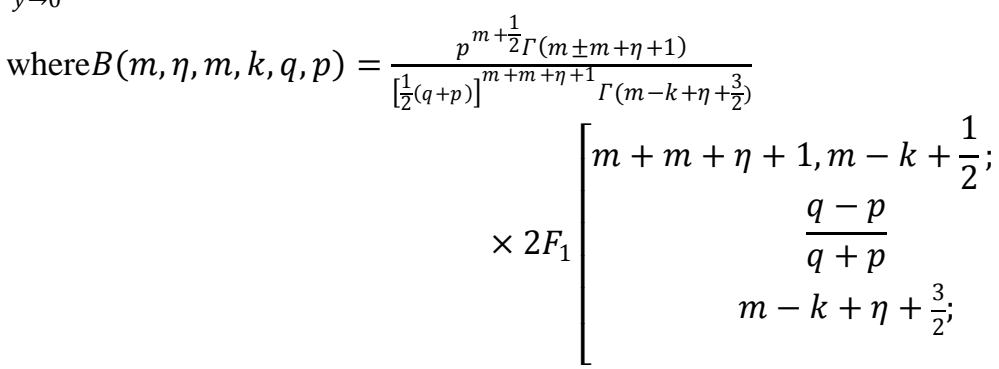

Proof: We prove the theorem for a restricted type of functions i.e. when $f(x, t)$ is seperable.

$$
\text { Let } f(x, t)=g(x) h(t) \quad(5.1)
$$

Therefore we can assume that $\beta=\beta_{1} \cdot \beta_{2}$, where $\beta_{1}, \beta_{2}$ are real numbers such that,

Property (ii) becomes

$$
\left.\begin{array}{cc}
\lim _{x \rightarrow \infty} \frac{g(x)}{\left(\log _{b}\right)^{v}}=\beta_{1} & \operatorname{Re} v>-1 \\
\lim _{t \rightarrow \infty} \frac{h(t)}{t^{\eta}}=\beta_{2} & \eta>-1
\end{array}\right\},
$$

We also know that $(M(g(x)))(s)=\int_{0}^{\infty} g(x) x^{s-1} d x$

Since $g(x)$ has compact support in $[b, \infty)$ we have, 
$=\int_{b}^{\infty} g(x) x^{s-1} d x$

If we put $x=b e^{V}$ in above equation

$=\int_{0}^{\infty} g\left(b e^{V}\right) b^{s} e^{s V} d V=b^{s} \int_{0}^{\infty} g\left(b e^{V}\right) e^{-e^{i \pi} s V} d V$

$=b^{s}\left(L g\left(b e^{V}\right)\right)\left(e^{i \pi} s\right)$.

Also for $\eta>-1$ and $t>0$

$\int_{0}^{\infty} t^{\eta} e^{-\frac{q}{2} y t}(y t)^{m-\frac{1}{2}} W_{k, m}(p y t) d t=\frac{1}{y^{\eta+1}} B(m, \eta, m, k, q, p)$

$\int_{0}^{\infty} V^{v} e^{s V} d V=\frac{\Gamma(v+1)}{(-1)^{v+1} s^{v+1}}$ for Rev $>-1$

Now consider,

$\left|s^{v+1} y^{\eta+1} F(s, y)-\beta b^{s} \Gamma(v+1) B(m, \eta, m, k, q, p)\right|$

$=\mid s^{v+1} y^{\eta+1} \int_{0}^{\infty} \int_{b}^{\infty} x^{s-1} e^{-\frac{q}{2} y t}(y t)^{m-\frac{1}{2}} W_{k, m}(p y t) g(x) h(t) d x d t-\beta_{1} \beta_{2} b^{s} \Gamma(v+1)$

$B(m, \eta, m, k, q, p) \mid(\because g(x)$ has compact support in $[b, \infty)$. $)(5.6)$

Put $x=b e^{V} \quad \therefore x^{s-1} d x=b^{s-1} e^{V(s-1)} \cdot b e^{V} d V=b^{s} e^{V} d V$

As $x$ varies from $b$ to $\infty$ and $V$ varies from 0 to $\infty$.

Using (5.4), (5.5) and the suggested substitution (5.6) becomes

$\left|s^{v+1} y^{\eta+1} F(s, y)-\beta b^{s} \Gamma(v+1) B(m, \eta, m, k, q, p)\right|$

$$
\begin{aligned}
& =\mid s^{v+1} y^{\eta+1} \int_{0}^{\infty} \int_{0}^{\infty} e^{-\frac{q}{2} y t}(y t)^{m-\frac{1}{2}} W_{k, m}(p y t) b^{s} e^{s V} g\left(b e^{V}\right) h(t) d V d t \\
& \quad-\beta_{1} \cdot \beta_{2}(-1)^{v+1} s^{v+1} b^{s} \int_{0}^{\infty} V^{v} e^{s V} d V y^{\eta+1} \int_{0}^{\infty} t^{\eta} e^{-\frac{q}{2} y t}(y t)^{m-\frac{1}{2}} W_{k, m}(p y t) d t \mid \\
& =\left|s^{v+1} y^{\eta+1} \int_{0}^{\infty} \int_{0}^{\infty} e^{-\frac{q}{2} y t}(y t)^{m-\frac{1}{2}} W_{k, m}(p y t) b^{s} e^{s V}\left[g\left(b e^{V}\right) h(t)-\beta_{1} \cdot \beta_{2} V^{v} t^{\eta}\right] d V d t\right| \\
& \leq I_{1}+I_{2}
\end{aligned}
$$

where

Consider

$$
\begin{aligned}
& I_{1}=\left|\begin{array}{l}
s^{v+1} y^{\eta+1} \int_{0}^{Y} \int_{0}^{Y} e^{-\frac{q}{2} y t}(y t)^{m-\frac{1}{2}} W_{k, m}(p y t) b^{s} e^{s V}\left[g\left(b e^{V}\right) h(t)-\beta_{1} \cdot \beta_{2} V^{v} t^{\eta}\right] d V d t \\
I_{2}=\mid s^{v+1} y^{\eta+1} \int_{Y}^{\infty} \int_{Y}^{\infty} e^{-\frac{q}{2} y t}(y t)^{m-\frac{1}{2}} W_{k, m}(p y t) b^{s} e^{s V}\left[g\left(b e^{V}\right) h(t)-\beta_{1} \cdot \beta_{2} V^{v} t^{\eta}\right] d V d t
\end{array}\right|
\end{aligned}
$$

$$
I_{1}=\left|s^{v+1} y^{\eta+1} \int_{0}^{Y} \int_{0}^{Y} e^{-\frac{q}{2} y t}(y t)^{m-\frac{1}{2}} W_{k, m}(p y t) b^{s} e^{s V} V^{v} t^{\eta}\left[\frac{g\left(b e^{V}\right) h(t)}{V^{v} t^{\eta}}-\beta_{1} \cdot \beta_{2}\right] d V d t\right|
$$

Using the asymptotic behaviour of $W_{k, m}(z)=O\left(z^{k} e^{-\frac{z}{2}}\right), \quad|z| \rightarrow \infty$

$$
\begin{array}{ccrl}
=O\left(z^{m+\frac{1}{2}}\right), & |z| & \rightarrow 0, \quad m<0 \\
= & O\left(z^{m-\frac{1}{2}}\right), & |z| \rightarrow 0, m>0
\end{array}
$$

As $\frac{f(x, t)}{x^{c}}=\frac{g(x) h(t)}{x^{c}}=\frac{g\left(b e^{V}\right) h(t)}{b^{c} e^{V c}}$ is absolutely integrable for some $c \in R$.

Therefore

Therefore $\frac{g\left(b e^{V}\right) h(t)}{e^{V c}}$ is absolutely integrable as $b^{c}$ is constant.

$$
I_{1}=\left|s^{v+1} y^{\eta+1} \int_{0}^{Y} \int_{0}^{Y} e^{-\frac{q}{2} y t}(y t)^{m-\frac{1}{2}} W_{k, m}(p y t) b^{s} e^{(s+c) V} V^{v} t^{\eta}\left[\frac{g\left(b e^{V}\right) h(t)}{V^{v} t^{\eta}}-\beta_{1} \cdot \beta_{2}\right] d V d t\right|
$$

Therefore $\int_{0}^{Y} \int_{0}^{Y} e^{-c V} V^{v} t^{\eta}\left[\frac{g\left(b e^{V}\right) h(t)}{V^{v} t^{\eta}}-\beta_{1} \cdot \beta_{2}\right] d V d t=M$

Let $\left(r_{1}, r_{2}\right)$ be the point where the r.h.s. function has upper bound $M$.

$\leq 0<V<Y\left|s^{v+1} y^{\eta+1} e^{-\frac{q}{2} y r_{2}}\left(y r_{2}\right)^{m-\frac{1}{2}} W_{k, m}\left(p y r_{2}\right) b^{s} e^{(s+c) r_{1}}\right| M$.

$$
0<t<Y
$$

Choose $y$ sufficiently small that r.h.s. $<\frac{\epsilon}{2}$.

Consider

$$
I_{2}=\left|s^{v+1} y^{\eta+1} \int_{Y}^{\infty} \int_{Y}^{\infty} e^{-\frac{q}{2} y t}(y t)^{m-\frac{1}{2}} W_{k, m}(p y t) b^{s} e^{s V}\left[g\left(b e^{V}\right) h(t)-\beta_{1} \cdot \beta_{2} V^{v} t^{\eta}\right] d V d t\right|
$$


$s^{v+1} y^{\eta+1} e^{-\frac{q}{2} y t}(y t)^{m-\frac{1}{2}} W_{k, m}(p y t) b^{s} e^{s V}$ is finite and continuous in $Y<t<\infty$ and $Y<x<\infty$ and it converges to zero as $t \rightarrow \infty$ and $V \rightarrow \infty$.

Therefore it is bounded (say) by $M$ as $t \rightarrow \infty$ and $V \rightarrow \infty$.

Now property (ii) $\Rightarrow \lim _{\substack{V \rightarrow \infty \\ t \rightarrow \infty}} \frac{g\left(b e^{V}\right) h(t)}{V^{v} t^{\eta}}=\beta_{1} \beta_{2}$

$\Rightarrow$ for all ( $\therefore$ for $\frac{\epsilon}{2 M}$ also ) there exists $M$ such that for $V>Y, t>Y$

$\therefore I_{2}<M \frac{\epsilon}{2 M}=\frac{\epsilon}{2}$

$$
\left|\frac{g\left(b e^{V}\right) h(t)}{V^{v} t^{\eta}}-\beta_{1} \beta_{2}\right|<\frac{\epsilon}{2 M}
$$

Using (5.8) and (5.9) statement (5.7) becomes

Hence the theorem.

$$
\left|s^{v+1} y^{\eta+1} F(s, y)-\beta b^{s} \Gamma(v+1) B(m, \eta, m, k, q, p)\right|<\epsilon
$$

\section{References:}

[1]. Bhosale, B. N. and Chaudhary, M. S.: Fourier-HankelTtansform of Distribution of compact support, J. Indian Acad. Math, 24(1), 2002, 169-190.

[2]. Sharma, V. D and Gudadhe, A. S.: Some Operators On Distributional Fourier-Mellin Transform, Science J.GVISH, 2, $2005,38-42$.

[3]. Kene, R. V. and Gudadhe, A. S.: On Distributional Generalized Mellin-Whittaker Transform, Aryabhata Research Journal of Physical Sciences, 12(5), 2009, 45-48.

[4]. Kene, R. V. and Gudadhe, A. S.: Some Properties of Generalized Mellin-Whittaker transform, Int. J. Contemp. Math. Sciences, 7(10), 2012, 477-488.

[5]. Zemanian, A. H.: Distribution Theory and Transform Analysis(McGraw-Hill, New York, 1965). 